\title{
ÍNDICE DE HIGIENE ORAL EN NIÑOS DE 6 AÑOS EN ECUADOR 2016
}

\section{INDEX OF ORAL HYGIENE IN 6 YEARS OLD CHILDREN, AT SAYAUSÍ ECUADOR 2016}

\author{
Vega-Ojeda Diego. ${ }^{1}$ \\ ${ }^{1}$ Odontólogo de la Universidad Católica de Cuenca.Ecuador. \\ *andres075@ hotmail.es
}

\begin{abstract}
Resumen
OBJETIVO: Calcular el Índice de Higiene Oral Simplificado comunitario en los escolares de 6 años de la parroquia Sayausi en el año 2016. MATERIALES Y MÉTODOS: La metodología aplicada fue de tipo cuantitativa, de diseño descriptivo, de técnica observacional y transversal actual. La muestra estuvo constituida por 158 pacientes de 6 años. El diagnóstico del examen bucal fue realizado por operadores que estuvieron calibrados bajo criterios del Índice de Higiene Oral Simplificado (IHO-S) de Greene y Vermillon. RESULTADOS: El nivel del Índice de Higiene Oral Simplificado obtenidos en la parroquia fue; $29 \%$ nivel excelente, $65 \%$ nivel bueno y $6 \%$ nivel regular. Los niveles de IHOS en pacientes de sexo masculino presentaron: $29 \%$ nivel excelente, $65 \%$ nivel bueno y $6 \%$ nivel regular, en el sexo femenino $29 \%$ nivel excelente, $64 \%$ nivel bueno y $7 \%$ nivel regular. CONCLUSIÓN: El nivel del Índice de Higiene Oral Simplificado comunitario en la parroquia es el $29 \%$ nivel excelente, $65 \%$ nivel bueno y un $6 \%$ nivel regular, mientras que el nivel de IHOS de acuerdo al sexo no existe diferencia significativa presentándose un mayor porcentaje el nivel bueno con el $65 \%$ en sexo masculino y $64 \%$ en el sexo masculino; el promedio de Placa Blanda en la parroquia es de 0,45; mientras el promedio de Placa Calcificada en la parroquia es del 0,02
\end{abstract}

Palabras clave: Índice de higiene bucal, índice de placa dental.

\begin{abstract}
AIM: Calculate the Community Simplified Oral Hygiene Index in 6 year olds from Sayausí in 2016. MATERIALS AND METHODS: A quantitative methodology, with a descriptive design of observational and transversal technique was applied. The sample consisted of 158 6-year-old patients. The diagnosis of the oral exam was performed by operators who were calibrated according to criteria of the Oral Hygiene Index (IHO-S) of Greene and Vermillon. RESULTS: The level of the Simplified Oral Hygiene Index obtained in the parish Sayausí was; $29 \%$ excellent level, $65 \%$ good level and 6\% regular level. The IHOS levels in male patients presented: 29\% excellent level, 65\% good level and 6\% regular level, in the female 29\% excellent level, 64\% good level and 7\% regular level. CONCLUSION: The level of the Community Simplified Oral Hygiene Index in the parish is $29 \%$ excellent level, $65 \%$ good level and $6 \%$ regular level, while there is no significant difference in the level of IHOS relating to sex presenting a higher percentage, level Good with $65 \%$ in males and $64 \%$ in males; The average plaque in the parish is 0.45; while the average Calcified Plate in the parish is 0.02 .
\end{abstract}

Key words: Oral hygiene index, dental plaque index.

\section{INTRODUCCIÓN}

En el presente trabajo se determinó y analizó el Índice de Higiene Oral Simplificado en los niños de 6 años de la parroquia Sayausi en el cantón Cuenca.

En los niños de edad escolar se observó una alta frecuencia de enfermedades bucodentales siendo uno de los principales factores causantes la placa bacteriana, entre las principales son la enfermedad periodontal y caries dental, las que ocasionan dolor, malestar al niño y repercusión en su calidad de vida. Greene y Vermillion crearon el Índice de Higiene Oral Simplificado (IHO) en el año de 1960 el mismo que presenta dos componentes: Índice de Detritus Simplificado (DI-S) y el Índice de Cálculo Simplificado (CI-S). El IHO se rige en determinaciones numéricas que representan la cantidad de restos o cálculo que se encuentra en las superficies de los dientes. Este método tiempo después fue modificado llamándose Índice de Higiene Oral Simplificado (IHOS) está conformado por los mismos componentes del 
original, diferenciados en que su aplicación se realiza solo en 6 superficies dentales preseleccionadas, 4 dientes anteriores y 2 posteriores, el cual constituye un método rápido para evaluar la limpieza bucal de los grupos poblacionales debido a que toma menos dientes y tiempo para su análisis.

La higiene bucal es un factor importante en la salud integral de la persona en cualquier etapa de la vida, el descuido o la falta de conocimiento de los niños en higiene oral provoca la acumulación de placa dentobacteriana, una de las principales causas que generan la aparición de la caries y enfermedad periodontal, debido a la aglomeración de una comunidad diversa de microorganismos los cuales se adhieren a las superficies dentales, está en ocasiones suele no ser visible, se forma después de consumir algún alimento siendo la población infantil las más vulnerable.

La enfermedad periodontal es la inflamación e infección que se disemina desde las encías hasta el ligamento periodontal y hueso que dan soporte al diente, la periodontitis es la causa principal de la caída de los dientes en los adultos.

El plan nacional de salud bucal está enfocado a la prevención y promoción, con el objetivo de prevenir las enfermedades y de mejorar su calidad de vida. La promoción de la Higiene oral y la prevención de enfermedades son temas de gran importancia en el campo de la salud pública, cuyo propósito es tener una relación con los escolares sobre el auto cuidado de su salud oral, buen punto de partida para alcanzar una correcta educación para la salud desarrollándose en las instituciones educativas con el ministerio de salud siendo la principal entidad responsable.

La Universidad Católica de Cuenca tiene entre sus funciones la investigación y la vinculación con la comunidad, en ese sentido asume vigencia y carácter urgente una descripción de la epidemiología de los factores de riesgo, sobre todo en las edades que recomienda la Organización Mundial de la Salud (OMS) que es son 6, 12 y 18 años, tratándose en esta oportunidad de una población de 6 años. Por lo cual se realizó la presente investigación epidemiológica sobre el Índice de Higiene Oral Simplificado en sector urbano en escuelas públicas como privadas.

\section{MATERIALES Y MÉTODOS}

La investigación fue de tipo cuantitativo, diseño descriptivo, técnica observacional y transversal actual, la muestra es de 158 escolares de 6 años, matriculados en las unidades educativas fiscales y particulares de la parroquia Sayausi, los mimos que cumplieron los criterios de inclusión (estudiantes matriculados en los centros educativos, consentimiento firmado por los padres, asentimiento del escolar) y de exclusión (enfermedades sistémicas, problemas de locomoción, alteraciones psicológicas, que falten el día del examen bucal y/o que no hayan aceptado el examen bucal). la muestra pertenece el $58 \%$ al sexo masculino y el $42 \%$ al sexo femenino. Los datos fueron tomados en el mes

\begin{tabular}{cc}
\hline CLASIFICACIÓN & PUNTUACIÓN. \\
\hline Excelente. & 0 \\
Buena. & $0.1-1.2$ \\
Regular. & $1.3-3.0$ \\
Mola. & $3.1-6.0$ \\
\hline
\end{tabular}

Tabla 1. Criterios para categorizar en niveles el IHOS.

de Enero del 2016 con la utilización de las fichas originales del Mapa Epidemiológico de Salud Bucal para la recolección de información la misma que está conformada por datos generales del paciente, Índice de Higiene Oral Simplificado, Índice de Caries CPOD, Índice de enfermedad Periodontal de Russel y mal oclusiones.

El examinador empezó observando con luz natural al niño sentado en una silla con la ayuda de un colaborador el cual iba anotando en la ficha, comenzaron observando las cara vestibular del el 1er molar superior derecho, continuando con el 1er incisivo superior derecho, luego 1er molar superior izquierdo. Luego empezará los inferiores, observando la cara lingual del el 1er molar inferior izquierdo, continuando con el 1er incisivo inferior izquierdo, luego ler molar inferior derecho. Si una de las piezas mencionadas no se encontraba, el código fue no registrable (NR)

Este procedimiento se realizó tanto para el componente de residuos blandos como para el de cálculo, obteniendo 2 resultados los cuales se sumaron y se obtuvo el IHO-S individual, una vez establecido, se procedió a determinar el grado clínico de higiene bucal:

El examinador debió seguir las siguientes recomendaciones:

a) En lo posible no tocar la boca del paciente con los dedos.

b) Inicialmente, cada diente lo examinó en forma visual.

c) Uso de un explorador solo en el caso que sea necesario.

d) Indagó al paciente la razón de la ausencia del diente, pero si la respuesta no le permitió obtener una conclusión, el examinador siguió su criterio clínico.

e) Procedió a dictar el código claramente para evitar errores de anotación.

f) Examinó todas las superficies del diente.

Para la aplicación de algunos criterios al momento del examen es aconsejable tener en mente la secuencia de erupción dentaria. Se considera como erupcionado un diente deciduo o permanente cuando cualquier porción de su corona clínica ha atravesado la fibro mucosa gingival y puede ser tocado con explorador dental. Para el registro de hallazgo los datos se consignan en las casillas correspondientes, anotando el código correspondiente que representa el criterio 
de clasificación o denominación del Hallazgo clínico, según los respectivos códigos que se detallan en los cuadros, para el análisis de datos se calculó la frecuencia de Índice de Higiene Oral Simplificado utilizando la fórmula de la misma.

\section{IHOS= Índice de Detritus + Índice de Cálculo.}

Luego se realizó el mismo cálculo pero agrupando por sexo y escuela. Finalmente se contó en cada individuo la cantidad de placa y cálculo dental para analizar el IHOS y se reportó como promedio poblacional general, por sexos y por cada Unidad Educativa. Estos valores fueron presentados en porcentajes.

\section{RESULTADOS}

La muestra estuvo distribuida de manera proporcional en los diferentes sexos (Gráfico 1). La muestra estuvo distribuida de manera no igualitaria siendo mayoritario en el sector fiscal (Gráfico 2). La muestra estuvo distribuida con un porcentaje elevado en el nivel bueno con el $65 \%$ (Gráfico $3)$. El promedio de IPB $(0,45)$ es mayor que el promedio de IPC $(0,02)$ en la parroquia Sayausí (Gráfico 4). La muestra estuvo distribuida de manera desigual, presentándose en el sector fiscal: $25 \%$ excelente, $67 \%$ bueno y $8 \%$ regular. En el particular presento: $48 \%$ excelente, $52 \%$ bueno y $0 \%$ regular (Gráfico 5). En el sexo femenino presenta un valor IPB menor que en el sexo masculino, mientras IPC en el sexo femenino es menor que en el sexo masculino (Gráfico 6). En el sexo masculino y femenino presentó un mayor porcentaje en el nivel bueno, mientras que en el sexo femenino un mayor porcentaje en el nivel regular (Gráfico 7).El sector fiscal presentó un Índice de Placa Blanda de 0,49; en el sector particular es de 0,29. En el sector fiscal el Índice de Placa Calcificada es de 0,03; en el sector particular es de 0,00. (Gráfico 8). La muestra estuvo distribuida de acuerdo al Índice de Higiene Oral Simplificado por cada unidad educativa donde la U.E Alborada presenta un promedio bajo en relación a la escuela Guardiana de la Fe que presenta un promedio alto (Gráfico 9). La mayor frecuencia en presentarse la Placa Blanda es en la pieza dental 1.6, y la menor frecuencia en Placa Blanda es en la pieza dental 7.1 (Gráfico 10). La muestra estuvo distribuida de acuerdo al Índice de Placa Calcificada por cada pieza dental presentándose en la pieza 1.6 con mayor cantidad PC, mientras que la Piezas 8.5-7.5 -7.1-3.1-1.1 ausencia de PC (Gráfico 11)

\section{DISCUSIÓN}

En cuanto al Índice de Higiene Oral Simplificado Valdivia, ${ }^{23}$ en el año 2016 en su estudio muestran valores de 1.55 IHOS comunitario que corresponde a un nivel regular. Fuentes y Cols. ${ }^{17}$ en el año 2014 presentaron en su estudio valores en el control pos-tratamiento un promedio de 0,94 de IHOS que corresponde a un nivel excelente. Rosas ${ }^{23}$ realizó

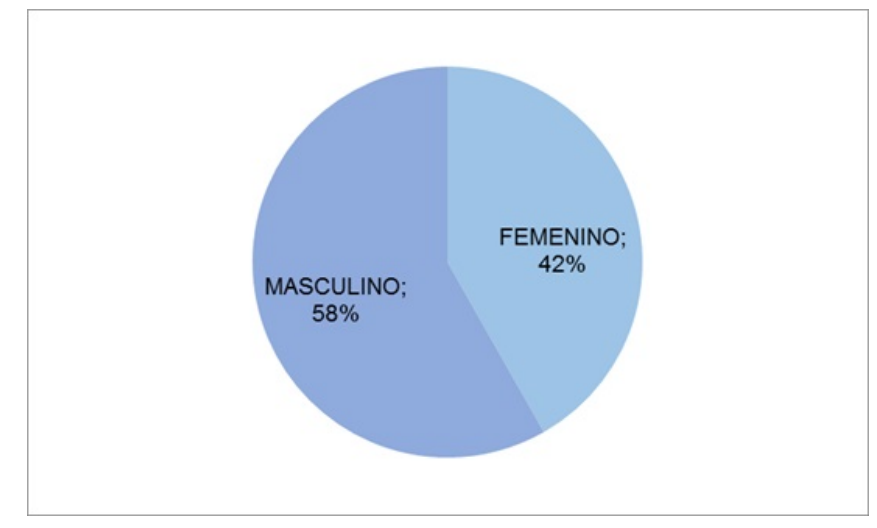

Grf. 1. Distribución de la muestra de acuerdo al sexo.

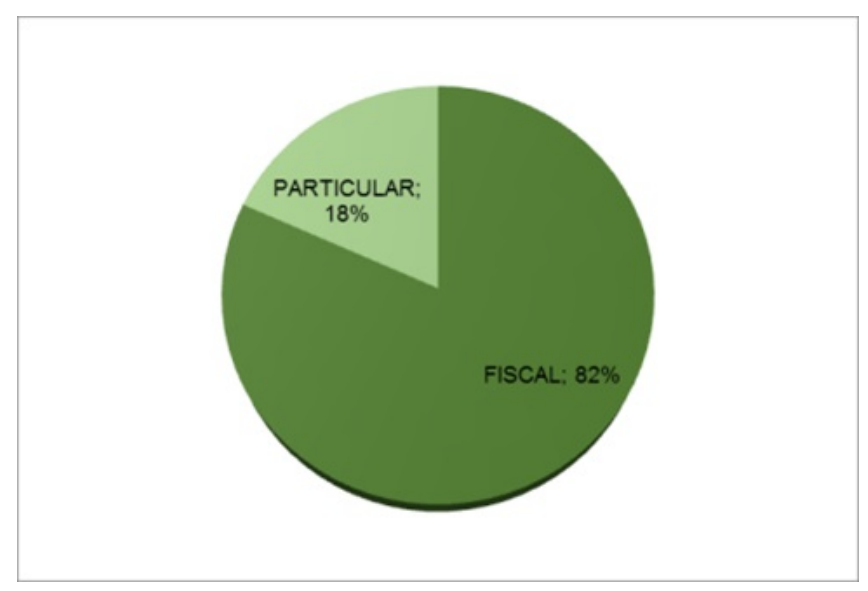

Grf. 2. Distribución de la muestra de acuerdo al tipo de gestión académica.

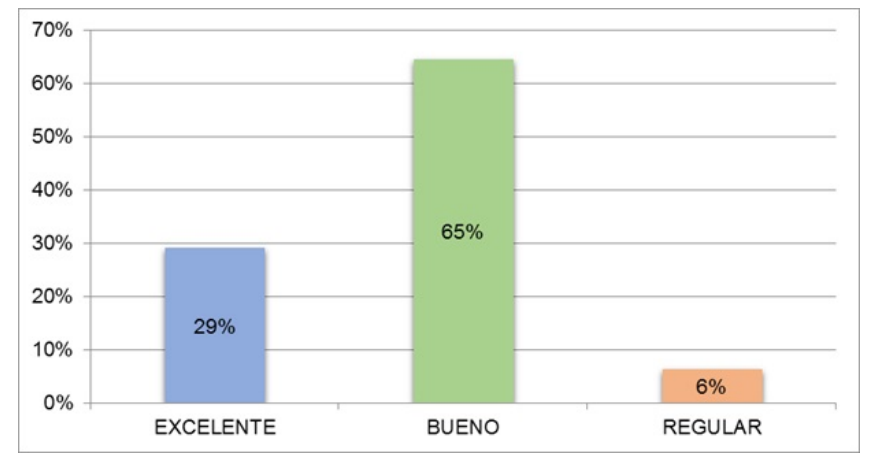

Grf. 3. Distribución de la muestra de acuerdo al nivel de Índice de Higiene Oral Simplificado de la parroquia Sayausí. 


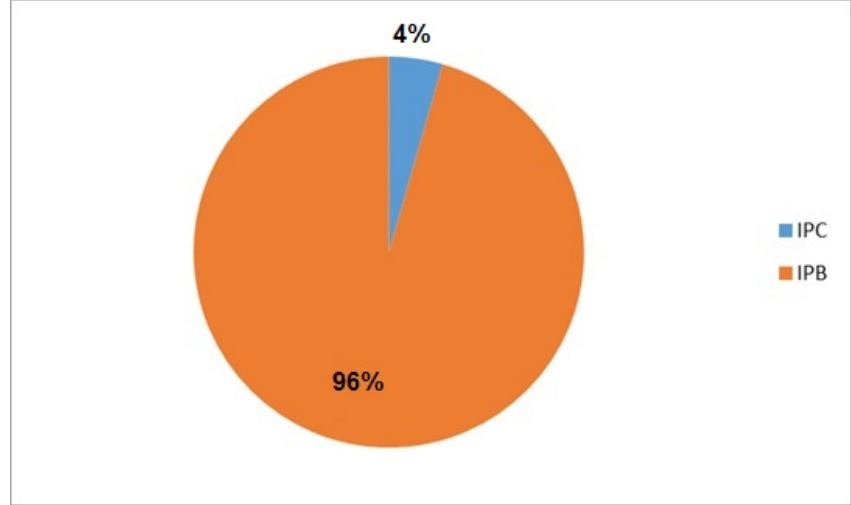

Grf. 4. Índice de Placa Blanda e Índice de Placa Calcificada de la parroquia Sayausí.

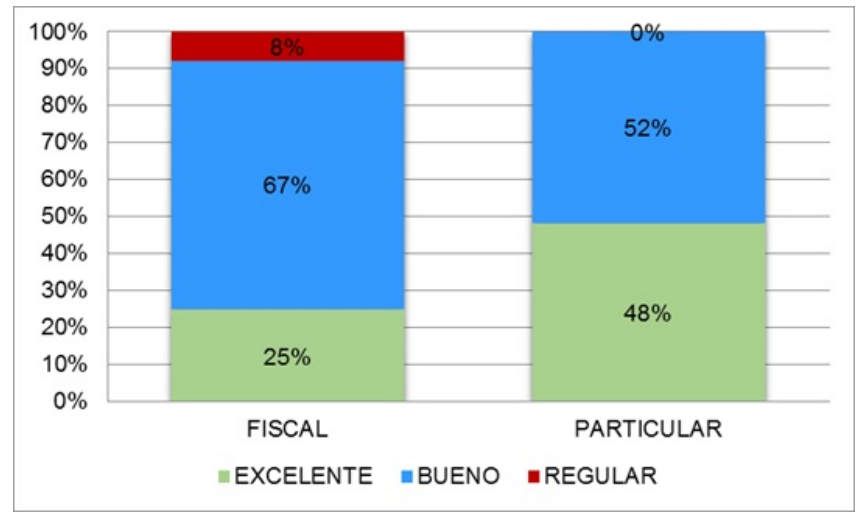

Grf. 5. Índice de Higiene Oral Simplificado según el tipo gestión académica.

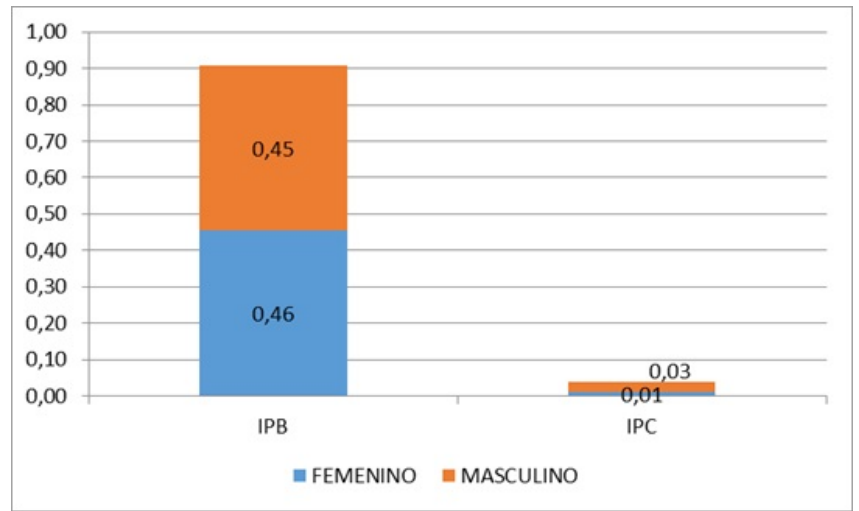

Grf. 6. Índice de Placa Blanda e Índice de Placa Calcificada según el sexo.

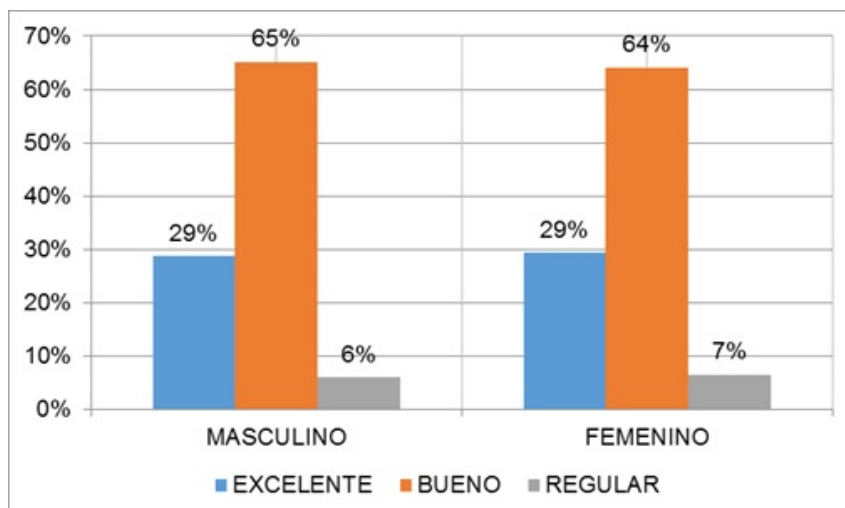

Grf. 7. Niveles del Índice de Higiene Oral Simplificado según el sexo.

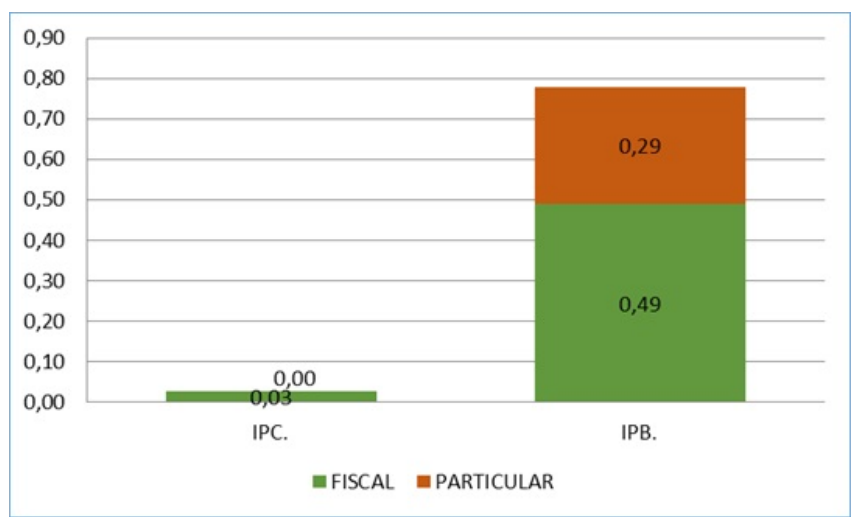

Grf. 8. Índice de Placa Blanda e Índice de Placa Calcificada según el tipo de gestión académica.

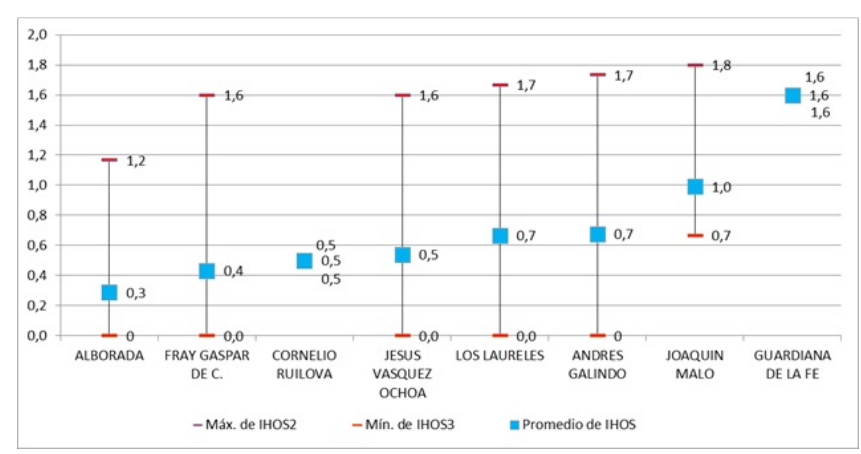

Grf. 9. IHOS comunitario en cada uno de los establecimientos educativos de la parroquia Sayausí. 


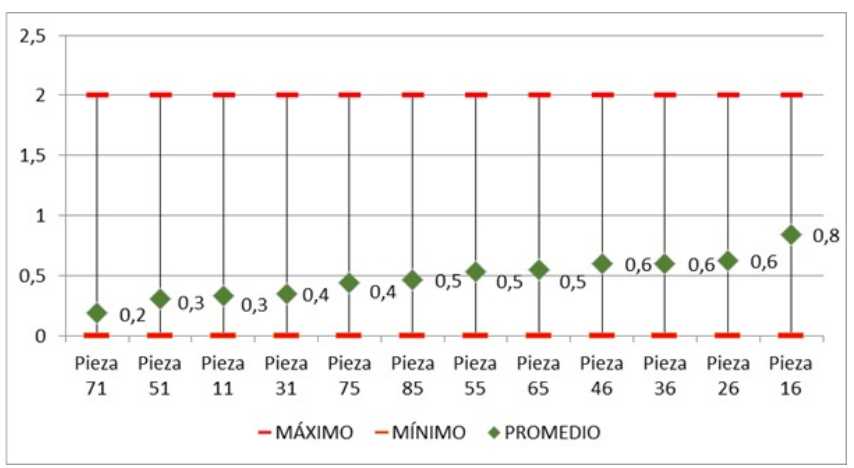

Grf. 10. Distribución de la muestra de acuerdo al promedio de IPB según la pieza dental.

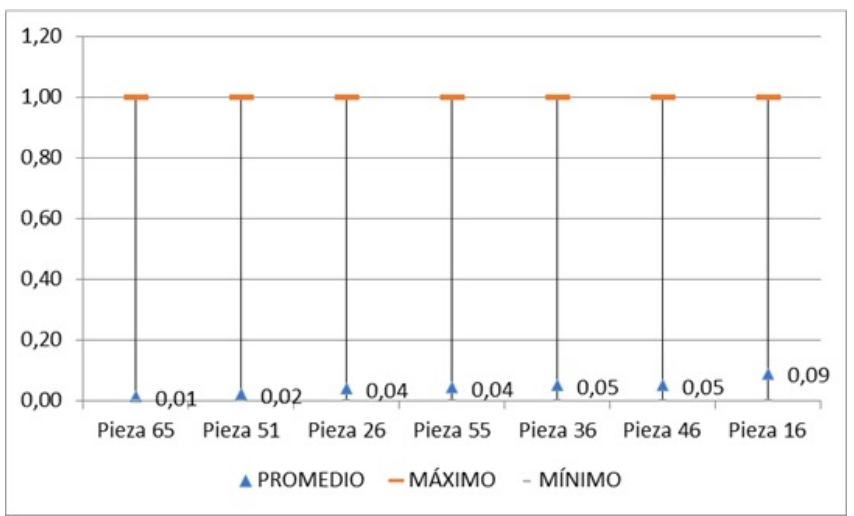

Grf. 11. Distribución de la muestra de acuerdo al promedio de IPC según la pieza dental.

un estudio similar en el año 2006, con niños de 6 a 12 años encontrando un valor promedio de 1,22 que corresponde a nivel regular. Moses ${ }^{4}$ en su estudio en niños de 6 a 12 años en el año 2014 presenta un 34,82\% con nivel bueno, mientras que el $57.49 \%$ presentaron higiene oral regular. En la parroquia Sayausí se obtuvo valores de manera no simétrica, presentándose: 102 individuos en el nivel BUENO de IHO-S que pertenece al $65 \%, 46$ individuos en el nivel excelente de IHO-S que pertenece al $29 \%$ y 10 individuos en el nivel regular de IHO-S que corresponde al $6 \%$, lo que diferencia de los resultados reportados la razón de la diferencia con estos estudios es el nivel socioeconómico, sector urbano y rural e infraestructura médica. Los resultados encontrados en los estudios muestran que existe un alto nivel de Índice de Higiene Oral Regular, no así como lo muestra esta investigación en donde los niños presentan un nivel de Índice de Higiene Oral Buena. En relación al nivel de Índice de Placa Blanda e Índice de Placa Calcificada Arpita $^{20}$ en el año 2006, en su estudio presentan el Índice de Placa Blanda $14.6 \%$ de nivel bueno de IPB, $65.9 \%$ de nivel regular de IPB y el $19.5 \%$ de nivel malo de IPB, el Índice de Placa Calcificada $91.9 \%$ de nivel bueno de IPC, $8.0 \%$ de nivel regular de IPC y el $0.1 \%$ de nivel malo de IPC. En el año 2006, Quisca ${ }^{19}$ en su investigación en escolares de 6 a 17 años encontró 1,30 de placa blanda y 0,16 de placa calcificada, en esta investigación la parroquia presenta un promedio de $\mathrm{O}, 45$ IPC y un promedio de 0,02 de IPC, estos datos son diferentes debido a que las condiciones socio culturales son diferentes entre ambos grupos poblacionales.

En relación al nivel de Índice de Higiene Oral de acuerdo al sexo, Moses ${ }^{4}$ en el año 2013, en el género masculino presentó una mayor proporción de higiene regular con un porcentaje de $63.36 \%$ que el género femenino; mientras que el género femenino presentaron buena higiene oral $38.80 \%$ y de mala higiene oral $10.34 \%$. en Sayausí se encontraron valores en el sexo masculino: 59 individuos que corresponde al $65 \%$ nivel bueno, 27 individuos que corresponde al $29 \%$ nivel excelente y 6 individuos que corresponde al $6 \%$ nivel regular. En el sexo femenino se obtuvo: 43 individuos que corresponde al $64 \%$ nivel bueno, 19 individuos que corresponde el $29 \%$ nivel excelente y 4 individuos que corresponde el $7 \%$ nivel regular.

El nivel de índice de Higiene Oral de acuerdo al tipo de gestión académica Ramos $\mathrm{L}$ y Naranjo $\mathrm{J}^{6}$ en el año 2011 en su estudio presenta valores de IHOS en la escuela Himmelman de 1,9 índice de placa y de 0,01 de Índice de cálculo y en la escuela Héroes de Cenepa tiene un IHOS de 1,4 de índice de placa y 0 en índice de cálculo, en Sayausi se encontraron valores en donde el establecimiento fiscal presenta: 87 individuos que corresponde al $25 \%$ nivel excelente, 32 individuos que corresponde al $67 \%$ nivel bueno y 10 individuos que corresponde al $8 \%$ nivel regular, en el establecimiento particular presento: $48 \%$ excelente, $52 \%$ bueno y $0 \%$ regular. dichos resultados no coinciden con los valores obtenidos. El promedio de Índice Higiene Oral Simplificado comunitario es de 0.47 IHOS Los resultados obtenidos de la investigación constituyen en una herramienta importante para tomar acciones para el mejoramiento de la salud pública y privada, con esta información podemos implementar programas con el objetivo de mejorar la calidad de salud bucal y evitar la aparición de enfermedades orales.

\section{CONCLUSIÓN}

La frecuencia de los niveles de IHO-S comunitario es del $29 \%$ excelente, $65 \%$ bueno y un $6 \%$ regular. El IHO-S no tiene asociación con el género de los niños/as determinándose que el Índice de Higiene Oral que más prevalece con $65 \%$ en el sexo masculino y $64 \%$ en el sexo femenino es el nivel bueno, seguido se presenta con el $29 \%$ en ambos sexos el nivel excelente y el $6 \%$ en el sexo masculino y $7 \%$ en el sexo femenino en el nivel regular, según el tipo de gestión académica se determinó que el nivel con mayor prevalencia es el nivel bueno con $67 \%$ en el sector fiscal y $52 \%$ en el sector particular, el nivel excelente es mayor en el sector particular con el $48 \%$ en relación con el sector fiscal que presenta $25 \%$. 


\section{Referencias}

1 Fernández C., Alvarez A., López G., Fernández A. Microbiología Oral. Microral [Internet]. 2012 [citado 27 Agosto 2016].Documento Disponible

2 Ochoa E., Roldan O., Ramírez B., Franco Ángela., Mejía Olga. Significados del autocuidado bucal para los escolares de instituciones educativas oficiales de Medellín. Revista Nacional de Odontología. [Internet]. 2011 [citado 25 Agosto 2016]. Volumen 7, Número 13. Documento Disponible

3 Ministerio de Salud Pública. Protocolos Odontológicos. Salud Bucal. Primera ed. Dirección Nacional de Normalización. Quito: MINSA; 2015 Documento Disponible

4 Moses A. Caries dental asociada al índice de higiene oral simplificado en niños de 6 a 12 años de una institución educativa pública del distrito de Ate - Vitarte en el año 2013 [Internet]. Universidad Peruana de Ciencias Aplicadas - UPC; 2014 [citad 2016 May 5]. Documento Disponible

5 Mediavilla. F. Determinación del Índice de Higiene Oral Simplificado en niños y niñas de 6 a 12 años de edad de la fundación Remar - Quito, en el mes de julio del año 2011 [cited 2016 oct 26]. Documento Disponible

6 Ortega J. Índices de IHOS en alumnos de nuevo ingreso de la Facultad de Odontología de la región poza rica -Tuxpan durante el esi-2011 [internet]. Universidad Veracruzana Facultad de Odontología zona poza Rica - Tuxpan. 2011 [cited 2016 oct 26]. Documento Disponible

7 Ramos L, Naranjo. J. Índice de higiene oral simplificado comparativo entre niños y niñas de 6 a 12 años de edad, de la escuela fiscal "Himmelman" y la unidad educativa particular "Héroes del Cenepa" del cantón Cayambe de la provincia de Pichincha, en el periodo 2010-2011.[internet]. Universidad Central del Ecuador-facultad de Odontología Unidad de Investigación y Postgrado. 2011. [cited 2016 oct 26].

8 Bastidas E. La placa bacteriana. Sistema e-Salud ESPOCH [Internet].2005. 3 [citado 26 Oct 2016] Documento Disponible

9 Greene J.G, Vermillion J. R. The simplified oral hygiene índex [Internet]. PubMed. 1964 Ene; vol (68); no. de pag. 25-31.

10 Barranca A. Técnicas de Higiene Oral. Uv [Internet]. Universidad Veracruzana Región Veracruz Salud Bucal. 2011[28 Agosto 2016]. Documento Disponible

11 Gil L., Aguilar A., Cañamás S., Ibáñez Ca. Sistemática de la higiene bucodental: el cepillado dental manual [Internet]. 2005 [30 sep. 2016]; Volumen [15]: 1-14. Documento Disponible

12 Ministerio de Salud Subsecretaría de Salud Pública. protocolo de cepillado y aplicación comunitaria de barniz de flúor para intervención en párvulos. minsal[Internet]. 2012 Documento Disponible

13 Serrano M., Posse J., Fernández J. manual de higiene oral para personas con discapacidad [Internet]. 2012 [30 sep.
2016].

14 Muñoz S. José. Higiene bucodental. Pastas dentífricas y enjuagues bucales. Elsevier [Internet]. 26 Oct 2015; 28 agosto 2016.

15 Villavicencio E. [Internet]. El tamaño muestra en tesis de post grado. El tamaño muestral en tesis de post grado. ¿cuantas personas debo encuestar? researchgate [Internet]. 2016 [citado 31 Ags 2016]; pag 1-4 Documento Disponible 16 Villavicencio E, et al. Pasos para la planificación de una investigación clínica. oactiva.ucacue.edu.ec. 2016; 1(1):1-5. Documento Disponible

17 Fuentes J., Corsini G., Bornhardt T., Ponce A., Ruiz F. Prevalencia de Caries y Nivel de Higiene Oral en Niños de 6 años Atendidos Bajo la Norma GES y el Modelo JUNAEB. SciELO [Internet]. 2014[31 Ags 2016]; 8(3):385391. Documento Disponible

18 Quisca H. Mapa epidemiológico de la salud bucal en escolares de 6 a 17 años de la provincia de Arequipa 2006 (índice de higiene oral en el distrito de alto selva alegre). Perú 2006[1 Sep. 2016].

19 Arpita J. Mapa epidemiológico de la salud bucal en escolares de 6 a 17 años de la provincia de Arequipa 2006 (índice de higiene oral de Greene y Vermillon en el distrito de Cayba). Perú 2006[1 Sep. 2016].

20 Dueñas J.. Índice de higiene oral en escolares de 6 a 17 años de ambos sexos del distrito Paucarpata, Arequipa 2006. Perú 2006[1 Sep. 2016].

21 Pinto T. Relación entre el índice de higiene oral simplificado de desechos di-s de Greene y Vermillon y la profundidad de sondaje periodontal en pacientes fumadores de la ASOC. benéfica "remar" y no fumadores de la clínica odontológica en la U.C.S.M- Arequipa. 2006”. Perú 2006[1 Sep. 2016].

22 Valdivia J. Mapa epidemiológico de Salud Bucal en escolares de 6 a 17 años de la Provincia de Arequipa 2006 (Índice de Higiene Oral en el Distrito Jacobo D. Hunter. Perú 2006[1 Sep. 2016].

23 Rosas B. índice de higiene oral simplificado de Green y Vermillon de 6 a 17 años de edad del distrito de cerro colorado 2006. Perú 2006[1 Sep. 2016].

Recibido: 10 de Abril de 2016

Aceptado: 25 de Abril de 2016 